\title{
US Department of Energy Planning meeting to form the CMSN Team: \\ Building a unified computational model for the resonant X-ray scattering of strongly correlated materials \\ Grant: FG02-07ER46385
}

Applicant/Institution: Northern Illinois University

Street Address/City/State/Zip: DeKalb, IL 60115

Principal Investigator: Michel van Veenendaal

Address: Department of Physics

Telephone Number: (815) 753-0667

Email: veenendaal@physics.niu.edu

DOE/Office of Science Program Office: Office of Basic Energy Sciences

DOE/Office of Science Technical Program Manager Contact:

Dr. Dale D. Koelling

Materials Sciences and Engineering Division

Office of Basic Energy Sciences

SC-22.2/Germantown Building, Room F-425A

U.S. Department of Energy

1000 Independence Avenue, S. W.

Washington, D.C. 20585-1290

E-Mail: Dale.Koelling@science.doe.gov

Phone: (301) 903-2187

Fax: (301) 903-9513 
The planning meeting was held May 21-23 2008 at Argonne National Laboratory (ANL). The purpose of the meeting was to establish a network on building computational model for resonant elastic and inelastic x-ray scattering. This course of action was recommended by program officer Dale Koelling after the initial submission of a proposal for a Computational Materials Science Network to Basic Energy Sciences. The meeting consisted of talks and discussion. At the end of the meeting three subgroups were formed. After the successful formation of the team, a new proposal was written which was funded by BES. Since this was a planning meeting there were no proceedings. The program and titles of talks are given below. 
Participants:

\section{U. S. participants}

Ken Ahn

Arun Bansil

Jim Freericks

New Jersey Institute of Technology

Mark Jarrell

Northeastern University

Bob Markiewicz

Georgetown University

University of Cincinatti

John Rehr

Northeastern University

Eric Shirley

University of Washington

Michel van Veenendaal

National Institute for Standards and Technology

Northern Illinois University/Argonne National Laboratory

International participants

Tom Devereaux

Sadamichi Maekawa

University of Waterloo, Canada

Tohoku University, Japan

George Sawatzky

University of British Columbia, Canada

Andrij Shvaika

Institute of Condensed Matter Physics, Ukraine

Jeroen van den Brink

University of Leiden, the Netherlands

\section{Experimental Participants}

Peter Abbamonte

Zahid Hasan

University of Illinois, Urbana Champaign

John Hill

Princeton University

Ben Larson

Brookhaven National Laboratory

Oak Ridge National Laboratory

\section{Time and location.}

The planning meeting was held May 21-23. At Argonne National Laboratory (ANL). 


\section{PLANNING MEETING PROGRAM}

Each talk will had 40 minute time slot of 25 minutes plus 15 minutes for discussion.

\section{DAY 1:}

8:00-8:30 Welcome, Goals of the planning meeting

8:30-10:30 Scientific talks $1,2,3$

10:30-11:00 Integrative Discussion

11:00-12:20 Talks 4,5

12:20-1:30 Lunch with Working Groups

1:30-3:30 Talks $6,7,8$

3:30-4:00 Integrative Discussion

4:00-6:00 Talks $9,10,11$

6:00-6:30 Integrative Discussion

6:30-8:30 Dinner and Working Groups

8:30-10:00 Working Groups

DAY 2:

8:00-8:30 Reports of the Working Groups

8:30-10:30 Talks $12,13,14$

10:30-11:00 Integrative Discussion

11:00-1:00 Talks 15,16,17,18

1:00-2:30 Lunch and Working Groups

2:30-4:00 Plenary Discussion on the organization of the teams. Tasks are defined and final teams are formed.

4.00-6.00 Team members got together to set up an action plan for the network, to establish the subjects each Collaborative Research Team (CRT) will focus on and to find a workable plan to optimize the collaborative aspect of the CRT.

6:00-8:00 Dinner and working CRT groups

8:00-9:30 CRT teams finalize their plans and timelines

DAY 3:

8:30-10:00 Reports of the detailed working plans of the CRTs

10:00-10:30 Integrative Discussion for final plans

10:30-12:00 Wrap up planning for new proposal for the CMSN

Afternoon: Meeting Adjournment 


\section{Titles of talks}

1. Jeroen van den Brink, New light on magnetic excitations: indirect resonant inelastic X-ray scattering on magnons

2. Bob Markiewicz, Mott gap collapse in electron-doped cuprates probed by RIXS

3. Ken Ahn, X-ray spectroscopy on transition metal oxides-- Probe for band structures and inhomogeneities

4. Mark Jarrell, Dynamical Cluster Quantum Monte Carlo simulations of the cuprates and how to relate these many body problems to resonant $\mathrm{x}$-ray scattering

5. Michel van Veenendaal, Resonant Inelastic X-ray Scattering in early transitionmetal compounds

6. Arun Bansil, Realistic Modeling of Resonant X-Ray Scattering Spectra of the Cuprates: Opportunities and Challenges

7. Jim Freericks, Many-body physics issues associated with the core-hole propagator and resonant inelastic $\mathrm{x}$-ray scattering

8. Eric Shirley, Density functional theory approaches to the Bethe-Salpeter equation and resonant $\mathrm{x}$-ray scattering

9. Tom Devereaux, K-edge RIXS in edge and corner sharing cuprates

10. Andrij Shvaika, Kadanoff-Baym-Keldysh techniques for determining the corehole propagator and associated charge vertices

11. Zahid Hasan, Charge excitations, collective modes and order in Low-D and doped Mott insulators probed by Elastic \& Inelastic Resonant X-ray Scattering

12. John Rehr, Multiple Scattering Approach to Inelastic X-ray Scattering

13. George Sawatzky, Resonant Elastic Soft-X-ray Scattering

14. Young-June Kim/John Hill, Recent experimental progress in Resonant Elastic and Inelastic X-ray Scattering

15. Ben Larson, Inelastic X-ray Scattering as a probe of the dynamic structure factor

16. Peter Abbamonte, Orbital and charge order studied by Resonant soft X-ray Scattering

17. Sadamichi Maekawa, Resonant Inelastic X-ray Scattering on undoped and doped Hubbard systems.

18. Aleksi Soininen, Recent Developments in Calculations and Analysis of Inelastic X-ray Scattering 